PROCEEDINGS OF THE

AMERICAN MATHEMATICAL SOCIETY

Volume 133, Number 5, Pages 1335-1341

S 0002-9939(04)07691-9

Article electronically published on December 6, 2004

\title{
EXT VANISHING AND INFINITE AUSLANDER-BUCHSBAUM
}

\author{
PETER JØRGENSEN
}

(Communicated by Martin Lorenz)

\begin{abstract}
A vanishing theorem is proved for Ext groups over non-commutative graded algebras. Along the way, an "infinite" version is proved of the non-commutative Auslander-Buchsbaum theorem.
\end{abstract}

\section{INTRODUCTION}

Let $R$ be a noetherian local commutative ring, and let $X$ be a finitely generated $R$-module of finite projective dimension. The classical Auslander-Buchsbaum theorem states

$$
\text { pd } X=\operatorname{depth} R-\operatorname{depth} X \text {. }
$$

This can also be phrased as an Ext vanishing theorem, namely, if $M$ is any $R$ module, then

$$
\operatorname{Ext}_{R}^{i}(X, M)=0 \text { for } i>\operatorname{depth} R-\operatorname{depth} X .
$$

A surprising variation of this is proved in [1]: Suppose that $R$ is complete in the $\mathfrak{m}$-adic topology. Then equation (II) remains true if $X$ is any $R$-module of finite projective dimension, provided $M$ is finitely generated. In other words, the condition of being finitely generated is shifted from $X$ to $M$.

In Theorem 2.3 below, this result will be generalized to the situation of a noncommutative noetherian $\mathbb{N}$-graded connected algebra.

The route goes through an "infinite" version of the non-commutative AuslanderBuchsbaum theorem, given in Theorem 1.4. This result is a substantial improvement of the original non-commutative Auslander-Buchsbaum theorem, as given in [3, thm. 3.2], in that the condition of dealing only with finitely generated modules is dropped.

The notation of this paper is standard and is already on record in several places such as 2] or 3]. So I will not say much, except that throughout, $k$ is a field, and $A$ is a noetherian $\mathbb{N}$-graded connected $k$-algebra. However, let me give one important word of caution: Everything in sight is graded. So for instance, $\mathrm{D}(A)$ stands for $\mathrm{D}(\mathrm{Gr} \mathrm{A})$, the derived category of the abelian category $\operatorname{Gr}(A)$ of $\mathbb{Z}$-graded $A$-left-modules and graded homomorphisms of degree zero.

Received by the editors June 10, 2003 and, in revised form, February 2, 2004.

2000 Mathematics Subject Classification. Primary 16E30, 16W50.

Key words and phrases. $k$-flat dimension, depth, infinite non-commutative AuslanderBuchsbaum theorem, Ext groups, vanishing theorem.

(C)2004 American Mathematical Society Reverts to public domain 28 years from publication 


\section{Auslander-Buchsbaum}

Definition 1.1. For $X$ in $\mathrm{D}(A)$, define the $k$-flat dimension by

$$
\operatorname{k.fd} X=-\inf k \stackrel{\mathrm{L}}{\otimes}{ }_{A} X \text {. }
$$

Remark 1.2. Using a minimal free resolution, it is easy to see that if the cohomology of $X$ is bounded and finitely generated, then

$$
\text { k.fd } X=\mathrm{fd} X=\operatorname{pd} X \text {, }
$$

where fd stands for flat dimension and pd stands for projective dimension.

In the following lemma, $\mathrm{D}^{\mathrm{b}}(A)$, the full subcategory of $\mathrm{D}(A)$ consisting of complexes with bounded cohomology, and $\mathrm{D}^{+}\left(A^{\mathrm{op}}\right)$, the full subcategory of $\mathrm{D}\left(A^{\mathrm{op}}\right)$ consisting of complexes whose cohomology vanishes in low cohomological degrees, are used.

Lemma 1.3. Let $X$ in $\mathrm{D}^{\mathrm{b}}(A)$ have $\mathrm{fd} X<\infty$, and let $T$ in $\mathrm{D}^{+}\left(A^{\mathrm{op}}\right)$ be so that $\mathrm{h}^{i}(T)$ is a graded torsion module for each $i$. Then

$$
\inf T \stackrel{\mathrm{L}}{\otimes}{ }_{A} X=\inf T-\operatorname{k.fd} X .
$$

Proof. Observe that $\mathrm{fd} X<\infty$ implies

$$
\inf k \stackrel{\mathrm{L}}{\otimes} X>-\infty
$$

hence

$$
\text { k.fd } X<\infty \text {. }
$$

If $T$ is zero, then $\inf T=\inf T \stackrel{\mathrm{L}}{\otimes}{ }_{A} X=\infty$, and then the inequality (3) implies that the lemma's equation trivially reads $\infty=\infty$. So for the rest of the proof let us assume that $T$ is non-zero and hence inf $T<\infty$. Note that $T$ is in $\mathrm{D}^{+}\left(A^{\mathrm{op}}\right)$, so $\inf T>-\infty$, so $\inf T$ is a finite number.

First, consider the special case where $T$ is concentrated in degree zero. Here $T$ is just a non-zero graded torsion $A$-right-module, and the lemma claims

$$
\inf T \stackrel{\mathrm{L}}{\otimes}_{A} X=-\operatorname{k.fd} X=\inf k \stackrel{\mathrm{L}}{\otimes}_{A} X .
$$

Let us start by showing more modestly that

$$
\inf T \stackrel{\mathrm{L}}{\otimes}_{A} X \geq \inf k \stackrel{\mathrm{L}}{\otimes}_{A} X .
$$

If $F$ is a flat resolution of $X$, then this amounts to

$$
\inf T \otimes_{A} F \geq \inf k \otimes_{A} F .
$$

To prove this, note that since $T$ is a graded torsion module, it is the colimit of the system

where

$$
T\langle 1\rangle \subseteq T\langle 2\rangle \subseteq \cdots
$$

$$
T\langle j\rangle=\left\{t \in T \mid A_{\geq j} t=0\right\} .
$$

Each quotient $T\langle j\rangle / T\langle j-1\rangle$ is annihilated by $A_{\geq 1}$ and so has the form $\coprod_{\alpha} k\left(\ell_{\alpha}\right)$, so there are short exact sequences of the form

$$
0 \rightarrow T\langle j-1\rangle \longrightarrow T\langle j\rangle \longrightarrow \coprod_{\alpha} k\left(\ell_{\alpha}\right) \rightarrow 0
$$


Tensoring such a sequence with $F$ gives a short exact sequence of complexes, because $F$ consists of graded flat modules. The corresponding cohomology long exact sequence consists of pieces

$$
\mathrm{h}^{i}\left(T\langle j-1\rangle \otimes_{A} F\right) \longrightarrow \mathrm{h}^{i}\left(T\langle j\rangle \otimes_{A} F\right) \longrightarrow \coprod_{\alpha} \mathrm{h}^{i}\left(k \otimes_{A} F\right)\left(\ell_{\alpha}\right) .
$$

Induction on $j$ now makes it clear that

$$
\mathrm{h}^{i}\left(k \otimes_{A} F\right)=0
$$

implies

$$
\mathrm{h}^{i}\left(T\langle j\rangle \otimes_{A} F\right)=0 \text { for each } j,
$$

and this further gives

$$
\mathrm{h}^{i}\left(T \otimes_{A} F\right) \cong \mathrm{h}^{i}\left(\operatorname{colim} T\langle j\rangle \otimes_{A} F\right) \cong \operatorname{colim~h}^{i}\left(T\langle j\rangle \otimes_{A} F\right)=0,
$$

so the inequality (6) follows, and hence, so does the inequality (5). Note that the proof even works for inf $k \stackrel{\mathrm{L}}{\otimes}{ }_{A} X=\infty$.

Let us now step this up to show equation (4). Note that if

$$
\text { inf } k \stackrel{\mathrm{L}}{\otimes}{ }_{A} X=\infty,
$$

then the inequality (5) forces

$$
\inf T \stackrel{\mathrm{L}}{\otimes}_{A} X=\infty,
$$

and so equation (4) holds.

So let us assume that

$$
\inf k \stackrel{\mathrm{L}}{\otimes}{ }_{A} X<\infty .
$$

Because of the inequality (2), it follows that inf $k \stackrel{\mathrm{L}}{\otimes}{ }_{A} X$ is a finite number. By the inequality (5), equation (4) will follow if it can be proved that

$$
\mathrm{h}^{\inf k \stackrel{\mathrm{L}}{\otimes}_{A} X}\left(T \stackrel{\mathrm{L}}{\otimes}{ }_{A} X\right) \neq 0 .
$$

But since $T$ is non-zero and graded torsion, there is a short exact sequence $0 \rightarrow$ $k(\ell) \longrightarrow T \longrightarrow \tilde{T} \rightarrow 0$ of graded $A$-right-modules. This gives a distinguished triangle $k(\ell) \longrightarrow T \longrightarrow \tilde{T} \longrightarrow$ in $\mathrm{D}\left(A^{\text {op }}\right)$, and tensoring with $X$ and taking the cohomology long exact sequence gives a sequence consisting of pieces

$$
\mathrm{h}^{i}\left(k(\ell) \stackrel{\mathrm{L}}{\otimes}{ }_{A} X\right) \longrightarrow \mathrm{h}^{i}\left(T \stackrel{\mathrm{L}}{\otimes}{ }_{A} X\right) \longrightarrow \mathrm{h}^{i}(\tilde{T} \stackrel{\mathrm{L}}{\otimes} A X) .
$$

Since $T$ is graded torsion, so is $\tilde{T}$. The inequality (5) applied to $\tilde{T}$ gives

$$
\mathrm{h}^{i}\left(\tilde{T} \stackrel{\mathrm{L}}{\otimes}_{A} X\right)=0 \text { for } i<\inf k \stackrel{\mathrm{L}}{\otimes}_{A} X .
$$

Hence there is a piece of the long exact sequence that reads

$$
0 \longrightarrow \mathrm{h}^{\inf k \stackrel{\mathrm{L}}{\otimes}{ }_{A} X}\left(k(\ell) \stackrel{\mathrm{L}}{\otimes}{ }_{A} X\right) \longrightarrow \mathrm{h}^{\mathrm{inf} k \stackrel{\mathrm{L}}{\otimes}{ }_{A} X}\left(T \stackrel{\mathrm{L}}{\otimes}{ }_{A} X\right),
$$

proving equation (7) and hence equation (4).

Secondly, consider the general case where $T$ is not necessarily concentrated in degree zero. There is a spectral sequence

$$
E_{2}^{p q}=\mathrm{h}^{p}\left(\mathrm{~h}^{q}(T) \stackrel{\mathrm{L}}{\otimes}{ }_{A} X\right) \Rightarrow \mathrm{h}^{p+q}\left(T \stackrel{\mathrm{L}}{\otimes}_{A} X\right),
$$


which can be obtained as the second usual spectral sequence of the double complex defined by $M^{p q}=T^{p} \otimes_{A} F^{q}$, where $F$ is a flat resolution of $X$; cf. [4, thm. 11.19]. The spectral sequence converges because fd $X<\infty$ implies that it is first quadrant up to shift. Now, $\mathrm{h}^{q}(T)$ is graded torsion for each $q$, so if $\mathrm{h}^{q}(T)$ is non-zero, then the special case of the lemma dealt with above applies to $\mathrm{h}^{q}(T) \stackrel{\mathrm{L}}{\otimes_{A}} X$ and shows that

$$
\inf \mathrm{h}^{q}(T) \stackrel{\mathrm{L}}{\otimes_{A}} X=-\operatorname{k.fd} X .
$$

There are now two cases. The first case is

$$
\text { k.fd } X=-\infty \text {. }
$$

Here equation (8) gives that if $\mathrm{h}^{q}(T)$ is non-zero, then $\inf \mathrm{h}^{q}(T) \stackrel{\mathrm{L}}{\otimes}{ }_{A} X=\infty$, that is, $\mathrm{h}^{p}\left(\mathrm{~h}^{q}(T) \stackrel{\mathrm{L}}{\otimes} A X\right)$ is zero for each $p$. Of course this also holds for $\mathrm{h}^{q}(T)$ equal to zero, and so in the spectral sequence, $E_{2}^{p q}$ is identically zero. Therefore the limit $\mathrm{h}^{p+q}(T \stackrel{\mathrm{L}}{\otimes} A)$ of the spectral sequence is also zero, so $T \stackrel{\mathrm{L}}{\otimes} A$ is zero, so

$$
\inf T \stackrel{\mathrm{L}}{\otimes}{ }_{A} X=\infty \text {. }
$$

But inf $T$ is a finite number, and combining this with equations (9) and (10) says that the lemma's equation reads

$$
\infty=(\text { a finite number })-(-\infty),
$$

which is true.

The second case is

$$
\text { k.fd } X>-\infty \text {. }
$$

Here equation (8) gives that if $\mathrm{h}^{q}(T)$ is non-zero, then $\mathrm{h}^{p}\left(\mathrm{~h}^{q}(T) \stackrel{\mathrm{L}}{\otimes_{A}} X\right)$ is non-zero for $p=-\operatorname{k.fd} X$, but zero for $p<-\operatorname{k.fd} X$. Of course, if $\mathrm{h}^{q}(T)$ is zero, then $\mathrm{h}^{p}\left(\mathrm{~h}^{q}(T) \stackrel{\mathrm{L}}{\otimes} A X\right)$ is zero for each $p$. So in the spectral sequence, $E_{2}^{p q}$ is non-zero for $p=-\operatorname{k.fd} X$ and $q=\inf T$, but zero for lower $p$ or $q$. Hence $E_{2}^{- \text {k.fd } X, \inf T}$ can be used in a standard corner argument which shows that the lowest non-zero term in the limit $\mathrm{h}^{p+q}\left(T \stackrel{\mathrm{L}}{\otimes}{ }_{A} X\right)$ of the spectral sequence has degree $p+q=-\operatorname{k.fd} X+\inf T$. Hence

$$
\inf T \stackrel{\mathrm{L}}{\otimes}_{A} X=-\operatorname{k.fd} X+\inf T,
$$

proving the lemma's equation.

Observe that in the following theorem and the rest of the paper, depth $A$ stands for the depth of $A$ viewed as a left-module over itself.

Theorem 1.4 (Infinite Auslander-Buchsbaum). Assume that A satisfies that each $\operatorname{Ext}_{A}^{i}(k, A)$ is a graded torsion A-right-module. Let $X$ in $\mathrm{D}^{\mathrm{b}}(A)$ have $\mathrm{fd} X<\infty$. Then

$$
\operatorname{depth} X=\operatorname{depth} A-\operatorname{k.fd} X \text {. }
$$

Proof. I have

$$
\operatorname{RHom}_{A}(k, X) \cong \operatorname{RHom}_{A}\left(k, A \stackrel{\mathrm{L}}{\otimes}{ }_{A} X\right) \cong \operatorname{RHom}_{A}(k, A) \stackrel{\mathrm{L}}{\otimes}{ }_{A} X,
$$

where the second $\cong$ holds by [3] prop. 2.1] because $X$ is in $\mathrm{D}^{\mathrm{b}}(A)$ and has $\mathrm{fd} X<\infty$. 
Thus

$$
\begin{aligned}
\operatorname{depth} X & =\inf \operatorname{RHom}_{A}(k, X) \\
& =\inf \operatorname{RHom}_{A}(k, A) \stackrel{\mathrm{L}}{\otimes_{A} X} \\
& \stackrel{(a)}{=} \inf \operatorname{RHom}_{A}(k, A)-\mathrm{k} \cdot f d X \\
& =\operatorname{depth} A-\operatorname{kifd} X
\end{aligned}
$$

where (a) is by Lemma 1.3. The lemma applies because $\operatorname{RHom}_{A}(k, A)$ is in $\mathrm{D}^{+}\left(A^{\mathrm{op}}\right)$, and has ${ }^{i} \operatorname{RHom}_{A}(k, A)=\operatorname{Ext}_{A}^{i}(k, A)$ a graded torsion $A$-right-module for each $i$ by assumption.

Remark 1.5. Theorem 1.4 even holds for $\operatorname{depth} A=\infty$, where the theorem states that $\operatorname{depth} X=\infty$.

On the other hand, suppose $\operatorname{depth} A<\infty$. Then it is easy to see that it makes sense to rearrange the equation in Theorem 1.4 as

$$
\text { k.fd } X=\operatorname{depth} A-\operatorname{depth} X \text {. }
$$

If the cohomology of $X$ is bounded and finitely generated, then the equation of Theorem 1.4 reads

$$
\operatorname{depth} X=\operatorname{depth} A-\operatorname{pd} X
$$

by Remark 1.2 This is the original non-commutative Auslander-Buchsbaum theorem, as proved in [3, thm. 3.2].

\section{Ext VANISHING}

Lemma 2.1. Assume that $A$ has $\operatorname{depth} A<\infty$ and satisfies that each $\operatorname{Ext}_{A}^{i}(k, A)$ is a graded torsion A-right-module.

Let $X$ in $\mathrm{D}^{\mathrm{b}}(A)$ have $\mathrm{fd} X<\infty$, and let $T$ in $\mathrm{D}^{+}\left(A^{\mathrm{op}}\right)$ be so that $\mathrm{h}^{i}(T)$ is a graded torsion module for each $i$. Then

$$
\inf T \stackrel{\mathrm{L}}{\otimes}{ }_{A} X=\inf T+\operatorname{depth} X-\operatorname{depth} A .
$$

Proof. Using Lemma 1.3 and Remark 1.5 gives

$$
\inf T \stackrel{\mathrm{L}}{\otimes}_{A} X=\inf T-\operatorname{k.fd} X=\inf T+\operatorname{depth} X-\operatorname{depth} A .
$$

In the following theorem, $\mathrm{D}_{\mathrm{fg}}^{-}(A)$, the full subcategory of $\mathrm{D}(A)$ consisting of complexes whose cohomology vanishes in high cohomological degrees and consists of finitely generated graded modules, is used.

Theorem 2.2. Assume that $A$ has $\operatorname{depth} A<\infty$ and satisfies that each $\operatorname{Ext}_{A}^{i}(k, A)$ is a graded torsion A-right-module.

Let $X$ in $\mathrm{D}^{\mathrm{b}}(A)$ have $\mathrm{fd} X<\infty$, and let $M$ be in $\mathrm{D}_{\mathrm{fg}}^{-}(A)$. Then

$$
\sup \operatorname{RHom}_{A}(X, M)=\sup M-\operatorname{depth} X+\operatorname{depth} A .
$$


Proof. It is easy to see that since $M$ is in $\mathrm{D}_{\mathrm{fg}}^{-}(A)$, the Matlis dual $M^{\prime}$ is in $\mathrm{D}^{+}\left(A^{\mathrm{op}}\right)$ and has $\mathrm{h}^{i}\left(M^{\prime}\right)$ a graded torsion module for each $i$. So

$$
\begin{aligned}
\sup \operatorname{RHom}_{A}(X, M) & =\sup \operatorname{RHom}_{A}\left(X, M^{\prime \prime}\right) \\
& \stackrel{(\text { a) }}{=} \\
= & \sup \left(\left(M^{\prime} \otimes_{A} X\right)^{\prime}\right) \\
= & -\inf M^{\prime} \stackrel{\mathrm{L}}{\otimes}_{A} X \\
& \stackrel{(\mathrm{b})}{=}-\inf M^{\prime}-\operatorname{depth} X+\operatorname{depth} A \\
= & \sup M-\operatorname{depth} X+\operatorname{depth} A,
\end{aligned}
$$

where (a) is by adjunction and (b) is by Lemma 2.1 .

The following is the special case of Theorem 2.2 where $X$ and $M$ are concentrated in degree zero, that is, where $X$ and $M$ are graded modules.

Theorem 2.3 (Ext vanishing). Assume that $A$ has $\operatorname{depth} A<\infty$ and satisfies that each $\operatorname{Ext}_{A}^{i}(k, A)$ is a graded torsion A-right-module.

Let $X$ in $\operatorname{Gr}(A)$ have $\operatorname{fd} X<\infty$, and let $M$ be in $\operatorname{gr}(A)$. Then

$$
\operatorname{Ext}_{A}^{i}(X, M)=0 \text { for } i>\operatorname{depth} A-\operatorname{depth} X .
$$

If depth $X<\infty$ and $M \neq 0$ also hold, then

$$
\operatorname{Ext}_{A}^{i}(X, M) \neq 0 \text { for } i=\operatorname{depth} A-\operatorname{depth} X \text {. }
$$

This says that for $\operatorname{fd} X<\infty$, the number $\operatorname{depth} A-\operatorname{depth} X$ plays the role of projective dimension of $X$, but only with respect to finitely generated graded modules $M$.

Of course, this fails when $M$ is general, as illustrated by the following example.

Example 2.4. Let $A$ be the polynomial algebra $k[x]$. Then the conditions of Theorem 2.3 are satisfied, and it is classical that $\operatorname{depth} A$ is 1 .

Let $X$ be $k\left[x, x^{-1}\right]$. Then $\operatorname{depth} X \geq 1$, because $X$ is a graded torsion free module, so depth $A-\operatorname{depth} X \leq 0$ and Theorem 2.3 gives

$$
\operatorname{Ext}_{A}^{i}(X, M)=0 \text { for } i>0
$$

for $M$ in $\operatorname{gr}(A)$.

However, this must fail when $M$ is general, for otherwise $X$ would be a projective object of $\operatorname{Gr}(A)$, which it is certainly not.

\section{ACKNOWLEDGEMENT}

I would like to thank the referee for some very useful remarks, and Anders Frankild for showing me [1].

\section{REFERENCES}

[1] A. Frankild, Vanishing of local homology, Math. Z. 244 (2003), 615-630. MR1992028 (2004d:13027)

[2] P. Jørgensen, Gorenstein homomorphisms of non-commutative rings, J. Algebra 211 (1999), 240-267. MR1656580 (2000c:16013) 
[3] _ Non-commutative graded homological identities, J. London Math. Soc. (2) 57 (1998), 336-350. MR:1644217 (99h:16010)

[4] J. J. Rotman, "An Introduction to Homological Algebra", Pure and Applied Mathematics, Vol. 85, Academic Press, New York, 1979. MR0538169 (80k:18001)

Department of Pure Mathematics, University of Leeds, Leeds LS2 9JT, United KingDOM

E-mail address: popjoerg@maths.leeds.ac.uk

$U R L$ : www.maths.leeds.ac.uk/ popjoerg 